\title{
Os efeitos da gamificação social no desempenho dos estudantes durante a quarentena da Covid-19
}

\author{
Isabelle Melo do Nascimento ${ }^{1}$, José Rocha do Amaral Neto ${ }^{1}$, Luiz Oliveira da Silva \\ Junior ${ }^{1}$, Thaíse Kelly de Lima Costa ${ }^{1}$, Wilk Oliveira ${ }^{2}$
}

${ }^{1}$ Departamento de Ciências Exatas - Universidade Federal da Paraíba (UFPB) - Rio Tinto, PB - Brasil

${ }^{2}$ Instituto de Ciência Matemáticas e de Computação (ICMC) - Universidade de São Paulo (USP) - São Carlos, SP - Brasil

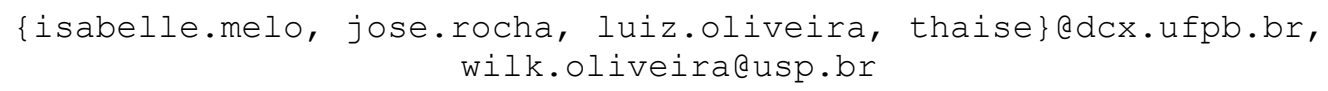

\begin{abstract}
Emergency remote teaching made teachers change their pedagogical practices, impacting students' social interactions. As a result, there was a need to apply new teaching techniques in order to rescue this interaction. In this sense, social gamification is a possibility, as it seeks to present only gamification elements that tend to impact social interaction. Therefore, we carried out a study with nine undergraduate students, seeking to identify the effects of social gamification on student performance. The results showed that this strategy alone is not enough to positively impact the user experience, making room for other types of gamification design.
\end{abstract}

Resumo. O ensino remoto emergencial fez com que os docentes mudassem suas práticas pedagógicas, impactando as interações sociais dos estudantes. Com isso, surgiu a necessidade de aplicar novas técnicas de ensino, a fim de resgatar essa interação. Nesse sentido, a gamificação social é uma possibilidade, visto que busca apresentar apenas elementos de gamificação que tendem a impactar a interação social. Diante disso, realizamos um estudo com nove estudantes de graduação, buscando identificar os efeitos da gamificação social no desempenho dos estudantes. Os resultados demonstraram que apenas tal estratégia não é suficiente para impactar de maneira positiva a experiência do usuário, abrindo espaço para outros tipos de design de gamificação.

\section{Introdução}

A adoção do ensino remoto emergencial, como medida preventiva e solução temporária para mitigar os efeitos causados pela pandemia da Covid-19 na educação, fez com que os docentes tivessem que repensar suas práticas pedagógicas no ambiente escolar [Bozkurt; Sharma 2020]. Para tanto, é válido destacar que a pandemia afetou milhões de estudantes, e cerca de metade dos universitários foram afetados com insegurança, medo, sensação de perda e mudanças de humor em comparação aos períodos letivos normais [Maia et al. 2020]. Diante disso, os atores da educação estão em busca de estratégias para despertar o interesse e motivação dos alunos no contexto atual [Rodrigues et al. 2021; Da Silva Costa et al. 2020].

Uma das soluções que está em ascensão, e já vem sendo utilizada há alguns anos 
como forma de enriquecer os ambientes de aprendizagem, é a gamificação (i.e., "o uso de elementos de jogos em contextos que não são jogos" [Deterding et al. 2011]) em educação [Koivisto, J. et al 2019]. Contudo, apesar de ser considerada uma técnica que produz resultados positivos, esta pode ter um impacto negativo na experiência do usuário, caso não seja aplicada de forma adequada [Toda et al. 2017]. Mediante isso, surge a personalização da gamificação, a qual compreende que as pessoas possuem perfis distintos e são motivadas de maneiras diferentes de acordo com seu perfil [Toda et al. 2019b; Oliveira et al. 2019]. Dentre os diferentes tipos de personalização da gamificação está a gamificação social, que busca apresentar apenas elementos de gamificação que visam impactar a interação social dos estudantes [Simões et al. 2013; De-Marcos et al. 2017; Toda et al. 2019a]. Sendo assim, a gamificação social pode ser usada como estratégia para melhorar o aspecto social e o desempenho dos estudantes.

Ao mesmo tempo, para que a gamificação personalizada seja aplicada na prática em sistemas educacionais é necessário identificar o perfil (user types) dos estudantes. Para a identificação desses perfis existem diferentes instrumentos, dos quais o principal do domínio de gamificação é a escala Hexad [Tondello et al. 2016]. A escala Hexad organiza os perfis dos usuários em seis, sendo eles: i) Socializers (usuários atraídos pelos aspectos sociais do jogo), que tendem a ser motivados por Relatedness (relacionamento com outras pessoas), e querem criar conexões sociais, ii) Philanthropists, que são motivados por um propósito, são altruístas e estão dispostos a dar sem esperar recompensa, iii) Free Spirits são motivados pela autonomia, pela liberdade para se expressar e agir sem controle externo, gostam de criar e explorar dentro de um sistema, iv) Achievers, por sua vez, são motivados pela competência, procuram progredir dentro de um sistema completando tarefas, ou provam seu valor ao enfrentar desafios difíceis, v) Players são motivados por recompensas extrínsecas, fazem de tudo para ganhar uma recompensa dentro de um sistema e vi) Disruptors se motivam pelo desencadeamento da mudança, gostam de testar os limites do sistema e tentar ir mais longe [Tondello et al. 2016].

Ademais, ao longo dos últimos anos, acadêmicos já vinham conduzindo estudos científicos que apontavam para vulnerabilidades relacionadas à saúde mental [Arino et al. 2018]. Consoante a isso, ao ser comparado o período normal ao pandêmico, foram encontradas diferenças estatisticamente significativas no que diz respeito aos níveis de ansiedade, depressão e estresse entre os estudantes [Estrela et al. 2020]. Dessa maneira, surge a necessidade de mensurar os efeitos da gamificação social, a fim de compreender em que grau esta pode ou não afetar o desempenho dos estudantes.

Com o objetivo de analisar o impacto provocado pela utilização da gamificação social no desempenho dos estudantes, neste artigo apresentamos os resultados de um estudo conduzido com nove estudantes de graduação em Licenciatura em Ciência da Computação, usando um sistema educacional gamificado. Este estudo ocorreu em três etapas: i) análise do perfil Hexad dos estudantes, ii) utilização do sistema (primeiro sem gamificação e depois com gamificação social), e iii) análise dos efeitos da gamificação no desempenho dos estudantes (em comparação com o sistema sem gamificação).

Os principais resultados obtidos no estudo trazem a tona que: i) a gamificação social teve um impacto positivo apenas nos perfis dominantes Free Spirit e Player, ii) o contexto em que o estudante está inserido deve ser levado em consideração, pois este pode impactar na experiência dos estudantes, iii) apenas o perfil dominante do estudante 
pode não ser suficiente para determinar o que impacta em seu desempenho. Nossos resultados contribuem para a comunidade de Informática na Educação, especialmente no que diz respeito ao design de estratégias gamificadas de ensino.

\section{Trabalhos Relacionados}

Esta seção será dedicada aos trabalhos que também se aprofundaram no tema abordado por esse estudo. Os critérios para seleção destes foram: $i$ ) publicação nos últimos anos $i$ ) ter abordado a temática da gamificação social em educação. Inicialmente, De-Marcos et al. (2017) utilizaram em seu estudo uma plataforma de gamificação social desenvolvida especialmente para aplicação em um curso de graduação com 15 semanas, que cobre os conceitos básicos de tecnologia da informação e comunicação, fornecendo conhecimentos e habilidades básicas de computação e aplicativos de escritório. Para tanto, 11 atividades remotas tiveram elementos de gamificação social incluídas em sua estrutura (duas a três atividades por módulo) e foi adotada a metodologia da avaliação em pares. Para fins de controle, os resultados foram comparados com os obtidos por outra turma, a qual usou o método de e-learning tradicional.

Por sua vez, Krause (2015) utilizou como objeto de estudos uma turma de estudantes de um curso que busca apresentar Python como uma linguagem de programação para análise estatística, e tem uma duração prevista de quatro semanas, comuma carga horária média de 4 horas por semana. O curso foi organizado em quatro aulas,cada aula contendo em média 15 videoaulas, e a retenção dos alunos foi medida com baseno número de vídeo aulas assistidas. Os alunos foram divididos em três grupos distintos, sendo estes um sem nenhum elemento gamificado, um com elementos de gamificação tradicionais e o terceiro com elementos de gamificação social. Os resultados apontaram que com a utilização da gamificação, a retenção teve uma média $25 \%$ maior, e esse aumento subiu para 55\% com a gamificação social. Igualmente, a precisão dos questionários teve aumento de $12,5 \%$ e $31 \%$, respectivamente, e a pontuação do teste finalteve aumentos de $22,5 \%$ com gamificação e $40 \%$ com gamificação social.

Finalmente, Mader (2019), utilizou um sistema de respostas do público com elementos de gamificação durante as aulas, onde os alunos foram divididos em equipes, e as respostas individuais somavam pontos para o time, sendo os resultados apresentados em tempo real para todos os participantes em seus laptops, bem como em uma tela da sala. Essas avaliações foram realizadas em dois cursos presenciais com diferentes configurações, sendo elas um curso que acompanha uma prática de desenvolvimento de software e um curso de lógica e matemática discreta, em que a gamificação foi usada para a segunda parte da aula sobre matemática discreta, sendo cada aluno designado aleatoriamente para uma das quatro equipes.

Em resumo, os trabalhos relacionados mostram que a gamificação social pode ser aplicada e trazer resultados satisfatórios, apesar de ressalvas relacionadas principalmente à forma como é aplicada. No entanto, denotam a falta de atenção a pontos específicos, como o contexto exclusivamente remoto de ensino, o fator pandêmico e a seleção dos elementos de gamificação. Assim, até onde sabemos, nosso estudo é o primeiro a avaliar os efeitos da gamificação social no desempenho dos estudantes durante o ensino remoto emergencial. 


\section{Design Experimental}

Esse estudo é de caráter quantitativo, usando dados reais de estudantes em um ambiente educacional gamificado personalizado.

\subsection{Objetivos e questões de pesquisa}

A presente pesquisa teve como objetivo analisar o impacto da gamificação social no desempenho de estudantes de uma disciplina de Estágio Supervisionado II, de um curso de Licenciatura em Ciência da Computação. Tal questionamento é pertinente, pois é possível perceber que, na aplicação da gamificação, diferentes perfis de usuários têm comportamentos distintos, dependendo do elemento de gamificação empregado e da atividade realizada, resultando em efeitos positivos ou negativos nas experiências dos usuários [Dichev; Dicheva 2017]. Diante disso, buscamos responder a seguinte questão de pesquisa: Como a gamificação social afeta o desempenho dos estudantes durante a quarentena da Covid-19?

\subsection{Materiais e metodo}

Na condução do estudo foi utilizada a ferramenta Eagle-edu ${ }^{1}$, um sistema educacional gamificado que permite aos docentes criarem turmas e aplicarem atividades, gamificadas ou não. Tal sistema foi escolhido por permitir que professores criem turmas sobre qualquer assunto, bem como personalizar a gamificação com base nas dimensões propostas por Toda et al. (2019a, b). Dessa maneira, foi possível usar o sistema com duas configurações diferentes (i.e., sem gamificação e com gamificação social). Os elementos de gamificação social utilizados no nosso estudo foram: Competição, Cooperação, Reputação e Pressão Social. Os elementos foram escolhidos com base no estudo de Toda et al. (2019), que define esses quatro elementos como sendo elementos sociais.

Foi utilizada a escala Hexad para identificar o perfil dos estudantes (user types), um modelo criado por Marczewski (2015) para auxiliar os designers a entender o tipo de pessoa que usa sistemas de jogo/gamificação. Para tanto, existe a representação de diferentes tipos de motivações e estilos de interação dos usuários [Marczewski 2015]. Essa escala pode identificar até seis user types, sendo eles: Socializer, Philanthropist, Free Spirit, Achiever, Player e Disruptor (ver detalhes sobre o perfil na Seção 1).

Com a finalidade de identificar o perfil Hexad dos estudantes utilizamos a escala que Tondello et al. (2016) propôs, a qual contém 24 questões. Visando evitar respostas de estudantes que estivessem sem atenção na hora de responder as questões da escala, incluímos uma "questão de checagem de atenção" (i.e., Essa é uma questão de checagem de atenção, se você leu essa questão, marque a opção 3), seguindo o exemplo de estudos recentes [Orji et al. 2018; Oliveira et al. 2020].

Para análise de dados, foram usados os softwares SPSS 27 (para análise estatísticas descritivas) e SmartPLS $3^{2}$ (para análise dos efeitos da gamificação social no desempenho dos estudantes) que provém uma interface para a realização de análises estatísticas baseadas em modelagem de equações estruturais (MEE) [Wong 2013]. A pesquisa foi organizada em três etapas: i) na primeira etapa, os estudantes responderam o Hexad. ii) na segunda etapa os estudantes usaram o sistema por duas semanas sem

\footnotetext{
1 https://eagle-edu.com.br/

2 https://www.smartpls.com/
} 
elementos de gamificação, e iii) na terceira etapa, os estudantes passaram a usar o sistema com gamificação social.

\subsection{Participantes e análise de dados}

A seleção da turma foi feita de maneira arbitrária, de acordo com a disponibilidade de disciplinas da Universidade onde o estudo foi realizado. Os alunos escolhidos foram os matriculados na turma de Estágio Supervisionado II, a qual é disponibilizada no $7^{\circ}$ período do curso. Esta conta com nove estudantes, oito do sexo masculino e um do sexo feminino. Devido a pandemia da Covid-19, esta disciplina não vinha sendo disponibilizada nos semestres anteriores, voltando a ser no primeiro semestre de 2021 .

Para analisar os efeitos da gamificação no desempenho dos estudantes, optamos por usar MEE de mínimos quadrados parciais (do Inglês, partial least squares structural equation modeling (PLS-SEM)) [Hair et al. 2016], uma técnica moderna robusta, que permite analisar as relações entre variáveis, com alto grau de confiabilidade mesmo em amostras muito pequenas que não podem ser analisadas mediantes outras técnicas [Hair et al. 2017].

\section{Resultados}

Inicialmente, a Tabela 1 apresenta os dados obtidos através do questionário Hexad. Quatro dos nove participantes tiveram apenas um perfil dominante (i.e., perfil Hexad com pontuação mais dominante), enquanto outros apresentaram mais de um perfil dominante (i.e., maior pontuação idêntica em mais de um perfil), como por exemplo, o estudante de ID 5 que possui três perfis (Achiever, Philanthropist e Socializer). Apenas este participante teve um desempenho maior com o design com gamificação social (DGS), do que o com design sem gamificação (DSG).

Tabela 1: Dados gerais

\begin{tabular}{c|c|c|c|c|c|c|c|c|c|c}
\hline ID & Sexo & $\mathrm{A}$ & $\mathrm{D}$ & $\mathrm{T}$ & $\mathrm{P}$ & $\mathrm{R}$ & $\mathrm{S}$ & Dominante & $\mathrm{DSG}$ & $\mathrm{DGS}$ \\
\hline 1 & $\mathrm{M}$ & 23 & 21 & 23 & 26 & 19 & 27 & $\mathrm{~S}$ & 15 & 11 \\
\hline 2 & $\mathrm{~F}$ & 25 & 13 & 26 & 27 & 14 & 16 & $\mathrm{P}$ & 15 & 13 \\
\hline 3 & $\mathrm{M}$ & 28 & 18 & 26 & 27 & 28 & 23 & $\mathrm{~A}$ and $\mathrm{R}$ & 15 & 13 \\
\hline 4 & $\mathrm{M}$ & 22 & 17 & 25 & 17 & 21 & 19 & $\mathrm{~T}$ & 15 & 14 \\
\hline 5 & $\mathrm{M}$ & 28 & 16 & 26 & 28 & 25 & 28 & $\mathrm{~A}, \mathrm{P}$ and S & 13 & 14 \\
\hline 6 & $\mathrm{M}$ & 28 & 9 & 27 & 27 & 25 & 28 & $\mathrm{~A} \mathrm{e} \mathrm{S}$ & 14 & 11 \\
\hline 7 & $\mathrm{M}$ & 23 & 15 & 24 & 24 & 22 & 20 & $\mathrm{~T} \mathrm{e} \mathrm{P}$ & 13 & 12 \\
\hline 8 & $\mathrm{M}$ & 24 & 11 & 23 & 23 & 18 & 19 & $\mathrm{~A}$ & 14 & 10 \\
\hline 9 & $\mathrm{M}$ & 20 & 18 & 20 & 18 & 19 & 17 & A e T & 15 & 12 \\
\hline
\end{tabular}


Legenda: M- Masculino; F- Feminino; A- Archiever; D- Disruptor; T- Free Spirit; P- Philanthropist; R- Player; S- Socializer; DSGDesign Sem Gamificação; DGS- Design Gamificação Social

Em seguida, com o intuito de assegurar a adequação dos dados à escala usada no estudo (i.e., escala Hexad), analisamos a confiabilidade e validade do constructo. Os resultados demostram que, com exceção das subescalas dos Disruptors e Free Spirits, os resultados foram adequados para todas as demais subescalas. Esses resultados estão organizados na Tabela 2 e são convergentes a outros estudos que também fizeram análises similares (e.g., Tondello et al. 2019; Santos et al. 2021).

Tabela 2: Confiabilidade e Validade do Constructo

\begin{tabular}{c|c|c|c|c}
\hline & $\begin{array}{c}\text { Cronbach’s } \\
\text { Alpha }\end{array}$ & $\begin{array}{c}\text { Jöreskog's } \\
\text { rho }\end{array}$ & $\begin{array}{c}\text { Confiabilidade } \\
\text { Composta }\end{array}$ & $\begin{array}{c}\text { Variância } \\
\text { Média } \\
\text { Extraída } \\
\text { (VME) }\end{array}$ \\
\hline Achiever & 0.765 & 0.846 & 0.805 & 0.528 \\
\hline Disruptor & 0.251 & -0.036 & 0.325 & 0.255 \\
\hline Free Spirit & 0.581 & 0.613 & 0.758 & 0.454 \\
\hline Philanthropist & 0.905 & 1.002 & 0.927 & 0.762 \\
\hline Player & 0.721 & 0.337 & 0.654 & 0.415 \\
\hline Socializer & 0.900 & 0.946 & 0.928 & 0.764 \\
\hline
\end{tabular}

Tabela 3: Matriz de correlação

\begin{tabular}{c|c|c}
\hline & DGS & DSG \\
\hline Achiever & -0.454 & 0.490 \\
\hline Disruptor & -0.070 & 1.013 \\
\hline Free Spirit & 0.801 & -0.081 \\
\hline Philanthropist & -0.027 & -0.520 \\
\hline Player & 0.642 & -0.351 \\
\hline Socializer & -0.685 & 0.407 \\
\hline
\end{tabular}


Por último, a Tabela 3 demonstra o efeito que cada design (i.e., sem gamificação e com gamificação) exerceu em relação a cada perfil. Podemos perceber que a gamificação social exerceu uma influência negativa em três dos seis perfis dos jogadores, sendo eles Achiever, Disruptor e Socializer. Outro ponto a se destacar é o do perfil Philanthropist, o qual teve influência negativa de ambos os designs de gamificação, porém com a gamificação social teve melhor influência.

Nesse estudo, optamos por não usar o método de bootstraping para análise de efeito, dado que o mesmo é sensível ao tamanho da amostra. Ao observar os dados obtidos, podemos perceber que os perfis foram impactados de diferentes formas. Os perfis Achiever, Disruptor e Socializer foram afetados negativamente com a gamificação social e positivamente sem elementos de gamificação. Em contrapartida, os perfis Free Spirit e Player foram impactados de maneira positiva com a gamificação social e negativa sem gamificação. No que diz respeito ao Philanthropist, este foi afetado de forma negativa em ambos os designs, porém com a gamificação social o impacto foi melhor.

\subsection{Discussões}

O final do ano de 2019 reservou para toda a sociedade um evento que viria a mudar completamente a forma como se lida com as mais diversas tarefas, entre elas, a educação. O despontar avassalador da pandemia da Covid-19 obrigou educadores do mundo todo a se adaptar a um cenário imediato de ensino remoto, o que propiciou diversas limitações e desafios. Falta de estrutura técnica, quadros psicológicos abalados, necessidade de novas habilidades, estas que deveriam ser aprendidas em tempo real para sua aplicação em sala de aula, fizeram com que novas estratégias se tornassem necessárias, e isso nos leva ao objeto de estudo deste trabalho, a gamificação social. O presente estudo buscou analisar o impacto de tal metodologia no aprendizado dos alunos, e ficou claro que ela pode ter reflexos positivos e negativos.

Inicialmente, os resultados do estudo revelaram que o perfil dominante que teve maior destaque foi o Achiever, em que os estudantes são motivados pela competência, buscando progredir dentro de um sistema, completando tarefas ou provando seu valor ao enfrentar desafios difíceis [Tondello et al. 2016]. De acordo com os resultados obtidos, foi possível observar que os perfis dominantes com maiores pontos de discrepâncias entre gamificação social e o design sem gamificação foram os Socializer e o Achiever. Sendo assim, os estudantes com esses perfis tiveram um melhor desempenho ao utilizarem a versão não gamificada do sistema.

Consoante a isso, mesmo os estudantes com perfil dominante Socializer foram influenciados de maneira negativa pela gamificação social, tendo um melhor desempenho ao utilizarem a plataforma sem gamificação. Para tanto, essa contradição detectada, em que os estudantes de perfis dominante Socializer tiveram um desempenho negativo em relação a gamificação social, pode ser justificado pelo impacto que o isolamento social causou nas interações sociais. Os estudantes que têm esses perfis são motivados por relatedness (relacionamento com outras pessoas). Estes querem interagir por meio de conexões sociais [Tondello et al. 2016]. Não obstante, a pandemia e a consequente adoção do ensino remoto fizeram com que as conexões destes alunos fossem impactadas, fazendo 
com que os elementos que possivelmente motivariam estes estudantes não tivessem o mesmo efeito.

Além disso, foi observado que a maior discrepância de desempenho em relação ao contexto com gamificação social e sem gamificação foi de quatro pontos a menos para a gamificação social. Para tanto, se torna intrigante o fato desses números terem sido obtidos por apenas dois participantes, de ID 1 e ID 8, em que o estudante de ID 1 tinha como perfil dominante Socializer. Nesse sentido, podemos considerar que o perfil dominante do participante não é suficiente para definir o resultado de sua experiência, sendo necessário analisar outros fatores, como por exemplo o contexto em que o estudante está inserido, confirmando os resultados de outros estudos (e.g., Hallifax et al. 2019).

O único estudante que obteve um melhor desempenho com a gamificação social foi o de ID 5, o qual possui três perfis dominantes (Achiever, Philanthropist e Socializer). É válido salientar que estudantes com o perfil dominante Philanthropist são motivados por propósitos [Tondello et al. 2016]. Diante disso, ele pode ter tido um melhor desempenho na gamificação social, uma vez que esta, diferentemente de um design sem gamificação, possui elementos que proporcionam um propósito ao estudante. Corroborando com essa perspectiva, a Tabela 3 mostra que o perfil Philanthropist teve um desempenho negativo tanto na gamificação social quanto no design sem gamificação, porém foi mais impactado negativamente sem nenhum elemento de gamificação. Nesse sentido, a discrepância dos resultados observados nas tabelas, pode estar intrinsecamente ligada ao impacto que o isolamento social causou.

Outro fator a ser destacado é que nenhum dos estudantes tiveram como perfil dominante o Disruptor, o qual se apresenta com maior incidência no ID 1. A menor diferença entre DSG e DGS, tendo predominância para DSG, é de um ponto, a qual está presente nos IDs 4 e 7, no qual ambos têm a presença do perfil Free Spirit. Destaca-se que os perfis que receberam melhor influência da gamificação social foram o Free Spirit e o Player, sendo o primeiro motivado pela autonomia, pela liberdade para se expressar e agir sem controle externo. Já o segundo é motivado por recompensas extrínsecas: ele faz de tudo para ganhar alguma recompensa dentro do sistema, independente do tipo da atividade [Tondello et al. 2016].

Em comparação com os resultados obtidos podemos perceber que, diferentemente da pesquisa realizada por De-Marcos et al. (2017), a nossa foi realizada com apenas um grupo de estudantes, e que estes tiveram a oportunidade de utilizar um sistema sem a gamificação e com a gamificação social. Ademais, também buscamos avaliar os perfis dominantes desse mesmo grupo. Diante disso, e considerando o atual contexto pandêmico, o qual deve ser levado em consideração na análise dos resultados, nosso estudo não comparou resultados com dois grupos diferentes e sim o impacto de atividades realizadas em um mesmo grupo de estudantes.

De-Marcos et al. (2017) destaca ainda que o grupo de gamificação social se saiu melhor nos pós-testes realizados. Porém, em uma análise geral, o grupo sem gamificação se saiu melhor. Ao comparar esses dados com os obtidos em nosso estudo percebemos que, de fato, os estudantes obtiveram um melhor desempenho na utilização do sistema sem gamificação. Não obstante, diferentemente do estudo realizado por De-Marcos et al. (2017), nossa pesquisa teve um fator externo a considerar, além de trazer implicações sobre a necessidade da aplicação de outros designs de gamificação, a fim de impactar positivamente estudantes de diversos perfis. Corroborando com isso, podemos salientar 
que o único estudante que obteve um melhor desempenho com a gamificação social foi o de ID 5, o qual possui três perfis dominantes (Achiever, Philanthropist e Socializer). Esse dado implica dizer que, não necessariamente, um aluno com determinado perfil só pode ser influenciado positivamente por elementos gamificados referentes àquele design de gamificação. Prova disso é o fato de um estudante com apenas o perfil Socializer ter tido um melhor desempenho ao utilizar o sistema sem a gamificação social.

Além do mais, os resultados obtidos com o estudo realizado por Krause (2015) mostraram que o grupo que utilizou gamificação simples teve um desempenho de retenção $25 \%$ melhor que o sem gamificação, e o grupo com gamificação social mais que dobrou esse resultado, atingindo 55\% mais retenção. Os testes aplicados offline também mostraram um ganho de $12,5 \%$ e $31 \%$, respectivamente. Mediante essa perspectiva, esse estudo mostra a discrepância entre os dados atingidos de maneira presencial e de maneira remota, os quais foram melhores no ensino presencial. Contudo, novamente, o estudo se distancia do presente trabalho pois, apesar de considerar a experiência offline, não considera o impacto do contexto pandêmico atual na saúde mental dos estudantes, e como isso afetou o desempenho destes. Ademais, Krause (2015) comprovou maior eficácia da gamificação social de maneira presencial do que de maneira remota.

Por fim, o trabalho de Mader (2019) traz implicações no que tange ao impacto de uma gamificação social baseada em equipe, em que esta teve uma influência positiva dependendo da configuração e do contexto de sua implantação. Em um pequeno curso com equipes pequenas, a gamificação social baseada em equipe teve um impacto positivo, enquanto em uma grande classe, com grandes equipes designadas aleatoriamente, a gamificação social baseada em equipe falhou em promover qualquer tipo de engajamento. Podemos citar como pontos discrepantes a diferença de contexto espacial, pois nosso trabalho foca em ensino remoto, bem como a duração de ambos.

Em síntese, pode-se destacar que em um quadro geral a gamificação social tende a propiciar resultados positivos, principalmente quando se observa seus efeitos em contexto presencial. Nesse sentido, nosso estudo reflete uma experiência não tão positiva por ter um fator pandêmico a considerar, bem como o consequente isolamento social que atingiu as interações sociais. Não obstante, traz implicações significativas, haja vista que levanta questionamentos sobre a importância da inclusão de outros designs de gamificação a fim de obter uma maior inclusão em termos de perfis dominantes dos estudantes, diferentemente dos estudos que já trabalharam com essa temática

\subsection{Limitações}

O contexto de ensino remoto tornou necessárias diversas adaptações, e todo processo de mudança traz consigo novos desafios e dificuldades, o que resultou em algumas limitações observadas no estudo. Inicialmente, foi necessário informar aos estudantes sempre sobre as tarefas, a fim de que estes não deixassem de fazê-las. Contudo, nem todos concluíram as atividades dentro dos prazos determinados, havendo ocorrências em que as tarefas acumularam e o aluno as resolveu de uma só vez, sem a estrutura proposta pelo estudo de semanas com e sem gamificação. Também foram observadas dificuldades técnicas, tais como uma ocorrência de autenticação, na qual uma discente não conseguiu recuperar sua senha, requisitando a criação de um novo perfil e exclusão do anterior. No intuito de minimizar essa limitação, em ambos os casos, excluímos dessas análises os dados de todos os participantes que não seguiram a estrutura proposta para o estudo. 
Houve também outras limitações relacionadas em sua maioria a interface da plataforma e seu comportamento, o qual apresentou alguns bugs e inconsistências na exibição do progresso dos estudantes. Ademais, os discentes usuários de smartphones notificaram dificuldades de navegação em partes específicas da plataforma. É válido também mencionar as limitações impostas pelo contexto pandêmico no que diz respeito a comunicação com os estudantes, dificultando a detecção de possíveis fatores externos de limitação de cada discente. Por fim, o tamanho de nossa amostra pode ser considerado pequeno para generalização dos resultados. No intuito de mitigar essa limitação, optamos por usar técnicas capazes de estimar resultados mesmo com amostras pequenas.

\subsection{Recomendações para pesquisas futuras}

Tendo em vista os resultados obtidos no estudo, e as limitações detectadas, foi possível obter algumas lições durante o processo de pesquisa, as quais geram oportunidades para outros estudos. Neste estudo foi utilizada a plataforma Eagle-edu, a qual possibilitou que fosse analisado o desempenho dos alunos sem nenhum tipo de gamificação e com a gamificação social. No entanto, a plataforma fornece outros tipos de designs de gamificação. Diante disso, sugerimos que: i) sejam realizadas replicações desse estudo com outros tipos de design de gamificação, ii) sejam realizadas análises similares em outros níveis de ensino, que possam avaliar novas experiências de usuários (e.g., motivação, ansiedade e equilíbrio), iii) sejam utilizados diferentes sistemas de medida e tratamento, para medir novas variáveis, ou seja, não só o desempenho e iv) sejam conduzidos estudos experimentais com amostras maiores.

\section{Considerações Finais}

Compreende-se que, no decorrer do período pandêmico, foram observadas alterações no bem-estar psíquico de estudantes universitários. Diante disso, esse quadro impactou negativamente a perspectiva destes estudantes. No estudo apresentado nesse artigo, os resultados mostram que, quando a plataforma estava sem elementos de gamificação, os estudantes tiveram um melhor desempenho do que quando foram utilizados elementos de gamificação social. Consoante a isso, pode-se destacar que somente a gamificação social não propicia resultados positivos, sendo necessário levar em consideração outros aspectos, como por exemplo o contexto pandêmico e outros designs de gamificação. Com estudos futuros tem-se o objetivo de replicar o estudo com um número maior de participantes e coletar dados relacionados à experiência destes, com a finalidade de realizar análises inferenciais, além de reproduzir com os outros tipos de perfil de gamificação. 


\section{References}

Arino DO, Bardagi MP. Relação entre Fatores Acadêmicos e a Saúde Mental de Estudantes Universitários. Psicol pesq. 2018.

Bozkurt, A.; Sharma, R. C. Emergency Remote Teaching in a Time of Global Crisis Due to Corona Virus Pandemic. Asian Journal of Distance Education, v. 15, i- vi,2020.

Da Silva Costa, Cássia Eufrásia et al. Aplicabilidade da gamificação em sala de aula em períodos de pandemia. Brazilian Journal of Development, v.6, n. 10, p. 79789- 79802, 2020 .

De-Marcos, Luis; Garcia-Cabot, Antonio; Garcia-Lopez, Eva. Towards the social gamification of e-learning: A practical experiment. International journal of engineering education, v. 33, n. 1, p. 66-73, 2017.

Deterding, S., Dixon, D., Khaled, R., \& Nacke, L. (2011). From game design elementsto gamefulness: defining" gamification". In Proceedings of the 15th international academic MindTrek conference: Envisioning future media environments (pp. 9- 15)

Dichev, C.; Dicheva, D. Gamifying education: what is known, what is believed and what remains uncertain: a critical review. International Journal of Educational Technology in Higher Education, [S.1.], v. 14, n. 1, p. 9, 2017.

Hair Jr, J. F., Hult, G. T. M., Ringle, C., \& Sarstedt, M. (2016). A primer on partial least squares structural equation modeling (PLS-SEM). Sage publications.

Hair Jr, J. F., Sarstedt, M., Ringle, C. M., \& Gudergan, S. P. (2017). Advanced issues in partial least squares structural equation modeling. saGe publications.

Hallifax, S., Serna, A., Marty, J. C., Lavoué, G., \& Lavoué, E. (2019, October). Factors to consider for tailored gamification. In Proceedings of the Annual Symposium on Computer-Human Interaction in Play (pp. 559-572).

Koivisto, J., \& Hamari, J. (2019). The rise of motivational information systems: A review of gamification research. International Journal of Information Management, 45, 191210.

Kocadere, Selay Arkün; Çaglar, Şeyma. Gamification from player type perspective: A case study. Journal of Educational Technology \& Society, v. 21, n. 3, p. 12-22, 2018.

Krause, Markus et al. A playful game changer: Fostering student retention in online education with social gamification. In: Proceedings of the Second (2015) ACM conference on Learning@ Scale. 2015.p.95-102.

Mader, Sebastian; BRY, François. Fun and engagement in lecture halls through social gamification. 2019

Maia, Berta Rodrigues; Dias, Paulo César. Anxiety, depression and stress in university students: the impact of COVID-19. Estudos de Psicologia (Campinas), v. 37, 2020.

Marczewski, Andrezej. User types. Even ninja monkeys like to play: Gamificiation, game thinking and motivational design, v. 1, p. 65-80, 2015.

Oliveira, Wilk; Bittencourt, Ig Ibert; Dermeval, Diego; Isotani, Seiji. Gamificação e Informática na Educação. In: Sampaio, Fábio F.; Pimentel, Mariano; Santos, Edméa 
O. (Org.). Informática na Educação: games, inteligência artificial, realidade virtual/aumentada e computação ubíqua. Porto Alegre: Sociedade Brasileira de Computação, 2021. (Série Informática na Educação, v.7) Disponível em: $<$ https://ieducacao.ceie-br.org/gamificacao/>

Oliveira, Wilk; Bittencourt, Ig Ibert. Tailored gamification to educational technologies. Springer Singapore, 2019.

Orji, Rita et al. Tailoring persuasive health games to gamer type. In: Proceedings of the sigchi conference on human factors in computing systems. 2013. p. 2467-2476.

Orji, R., Tondello, G. F., \& Nacke, L. E. (2018, April). Personalizing persuasive strategies in gameful systems to gamification user types. In Proceedings of the $2018 \mathrm{CHI}$ Conference on Human Factors in Computing Systems (pp. 1-14).

Ribeiro, Ralph Breno Silva et al. Investigação empírica sobre os efeitos da gamificação de um juiz online em uma disciplina de introdução à programação. Revista Brasileira de Informática na Educação, v. 28, p. 461-490, 2020.

Rodrigues, L., Toda, A. M., Oliveira, W., Palomino, P. T., Avila-Santos, A. P., \& Isotani,

S. (2021, March). Gamification Works, but How and to Whom? An Experimental Study in the Context of Programming Lessons. In Proceedings of the 52nd ACM Technical Symposium on Computer Science Education (pp. 184-190).

Santos, A. C. G., Oliveira, W., Hamari, J., Rodrigues, L., Toda, A. M., Palomino, P. T., \& Isotani, S. (2021). The relationship between user types and gamification designs. User Modeling and User-Adapted Interaction.

Simões, Jorge; Redondo, Rebeca Díaz; Vilas, Ana Fernández. A social gamification framework for a K-6 learning platform. Computers in Human Behavior, v. 29, n. 2, p. 345-353, 2013

Smiderle, Rodrigo. O efeito da gamificação no engajamento e aprendizagem de programação: um estudo considerado a personalidade e a orientação motivacional dos estudantes. 2019.

Smiderle, Rodrigo; Rigo, Sandro; Jaques, Patricia. Estudando o impacto da gamificação na aprendizagem e engajamento de alunos de acordo com os traços de personalidade e a orientação motivacional. In: Brazilian Symposium on Computers in Education (Simpósio Brasileiro de Informática na Educação-SBIE). 2019. p. 793.

Toda, A. M., Valle, P. H., \& Isotani, S. (2017). The dark side of gamification: An overview of negative effects of gamification in education. In Researcher links workshop: higher education for all (pp. 143-156). Springer, Cham.

Tondello, Gustavo F. et al. The gamification user types hexad scale. In: Proceedings of the 2016 annual symposium on computer-human interaction in play. 2016. P.229-243

Tondello, G. F., Mora, A., Marczewski, A., \& Nacke, L. E. (2019). Empirical validation of the gamification user types hexad scale in English and Spanish. International Journal of Human-Computer Studies, 127, 95-111.

Wong, K. K. K. (2013). Partial least squares structural equation modeling (PLS-SEM) techniques using SmartPLS. Marketing Bulletin, 24(1), 1-32. 